\title{
Les sept caractères et les quatre tempéraments dans Jules César de Shakespeare
}

\section{Jean Richer}

Marie-Thérèse Jones-Davies (éd.)

\section{(2) OpenEdition \\ Journals}

\section{Édition électronique}

URL : http://journals.openedition.org/shakespeare/439

DOI : 10.4000/shakespeare.439

ISSN : 2271-6424

\section{Éditeur}

Société Française Shakespeare

Édition imprimée

Date de publication : 1 novembre 1981

Pagination : 79-98

ISBN : 2-86433-016-4

Référence électronique

Jean Richer, "Les sept caractères et les quatre tempéraments dans Jules César de Shakespeare ", Actes des congrès de la Société française Shakespeare [En ligne], 3 | 1981, mis en ligne le 01 novembre 2007, consulté le 20 janvier 2020. URL : http://journals.openedition.org/shakespeare/439 ; DOI : $10.4000 /$ shakespeare.439 


\title{
THEATRE ET IDEOLOGIES :
} Marlowe, Shakespeare

\author{
DIRECTEUR DE LA PUBLICATION \\ M.T. Jones - Davies
}

JEAN TOUZOT Libraire - Editeur

38 , rue Saint-Sulpice 75278 PARIS CEDEX 061982 
1982 Jean Touzot Libiaire-Editeur, Paris.

Reproduction et traduction mzme partielle interdite.

Tous droits réservts potir lous pays

y compris i'U,R.S.S. et les pays scandinaves

ISBN 2-86433-016-4 


\title{
AVANT-PROPOS
}

L'opposition soulignée par Horace entre éthique et esthétique Aut prodesse volunt aut delectare poetae - que les Elisabéthains aiment à évoquer, pourrait être le lien qui rattache les unes aux autres les communications, rencontres et table ronde de notre troisième congrès (1981) sur le thème : "Théátre et Idéologies: Marlowe, Shakespeare.» Toutefois, le résultat de nos échanges montre un dépassement possible de l'alternative éthique / esthétique, puisqu'il met en valeur l'insertion d'idéologies susceptibles de transformer la fonction même de la littérature.

Les pièces analysées dans le contexte de leur temps révèlent de multiples aspects de la vision du monde et de la place que l'homme $y$ occupe, tels que les projette le théâtre anglais au tournant du XVIe et au début du XVIIe siècle.

Une exploration du langage et de la pensée des deux grands dramaturges aboutit à un commentaire sur les courants philosophiques ou religieux, sur les idées parfois contradictoires et sur la curiosité intellectuelle jamais assouvie qui se font jour à travers la rhétorique de la scène et le jeu dramatique, du Faust de Marlowe à La Tempéte de Shakespeare.

\author{
M.T. jones-Davies
}

Présidente de la Société Française Shakespeare 
SOCIETE FRANCAISE SHAKESPEARE Actes de Congrès.1981

\section{THEATRE ET IDEOLOGIES : Marlowe, Shakespeare}

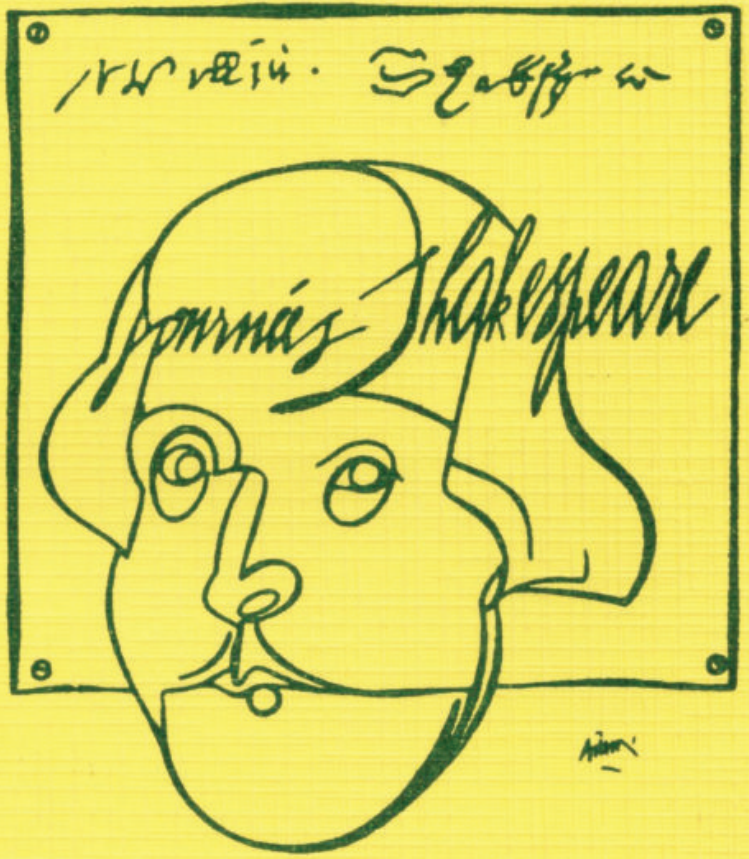

DIRECTEUR DE LA PUBLICATION M.T. Jones - Davies

JEAN TOUZOT Libraire - Editeur 38 , rue Saint-Sulpice 75278 PARIS CEDEX 061982 
Illustration de la couverture : affiche de Valerio Adami, spécialement conçue pour les Journées Shakespeare 1979 au Centre Georges Pompidou 


\section{TABLE DES MATIERES}

M.T. JONES-DAVIES Avant-propos

Théâtre et Idéologies: Marlowe, Shakespeare

S. TRUCHET

The Alchemy of Beauty: aesthetic principles in I Tamburlaine and their relationship to the hermetic ideology.

D. PRUDHOMME

L'idéologie du Juif de Malte et du Marchand de Venise.

D. GOY-BLANQUET

De Hall à Shakespeare : quelques glissements idéologiques opérés par la dramatisation dans Henry $I V$.

M.T. JONES-DAVIES Le Monıde du «si»: Idéologies Incertaines dans Comme il vous Plaira.

G. VENET

Temps et Idéologie : Marlowe, Shakespeare.

J. RICHER

Les Sept Caractères et les Quatre Tempéraments dans Jules César de Shakespeare.

F. LAROQUE

En marge de l'Idéologie : Antimasque et Grotesque dans le Dr Faustus et La Tempête.

A. LECERCLE-SWEET Conscience et Méconnaissance dans Edward The Second de Marlowe.

M.A. CONEJERO Shakespeare's Aesthe tic Scheme. (Extrait).

P.STEWART

Shylock, Shakespeare's Alien.

(Royal Shakespeare Company)

J.RUSSELL BROWN The Romantics' Shakespe are.

R. GILL

The Christian Ideology of Dr Faustus.

J. JACQUOT

Marlowe : de quelques problèmes d'interprétation. 
Rencontres entre Universitaires et Gens de Théâtre

Vendredi 4 décembre 1981
- Stuart Seide :Présentation du

Songe d'une nuit d'Eté. Mise en scène pour le Théâtre de Chaillot janvier 1982

- A propos d'Edouard (II) de

Bernard Turle

Bernard Turle : Métamorphoses de l'auteur et de l'écriture dramatiques. Intervention de Colin Harris (metteur en scène) 


\section{LES SEPT CARACTERES ET LES QUATRE TEMPERAMENTS DANS JULES CESAR DE SHAKESPEARE}

Dans l'introduction de l'édition Arden de Jules César T.S. Dorsch fait état d'une remarque de John Crow concernant l'abondance des allusions aux métaux dans cette pièce ' 1 . Mais il n'a pas vu qu'une telle symbolique n'était que la transposition dans un registre particulier de la symbolique des sept planètes traditionnelles. Comme nous allons l'établir dans cette pièce, que l'on date de 1599 , le dramaturge a concu les principaux personnages en termes de symbolique planétaire et, dans cette perspective particulière a tiré parti très habilement d'éléments qu'il trouvait dans Plutarque ${ }^{2}$.

\section{1- Antoine ou Mercure en Cancer :}

Dès la seconde scène du premier acte, Brutus déclare :

Je n'aime point les jeux : pour moi : je manque

De la vivacité qu'on voit en Marc-Antoine ${ }^{3}$

$$
\text { I, ii, 27-28. }
$$

Le texte anglais parle de "quick spirit», ce qui revient à attribuer à Marc- Antoine le «quick silver» ou mercure, donc à faire de lui le représentant du caractère mercurien, réputé fort prompt.

Losque les conjurés délibèrent pour savoir s'ils vont également tuer Antoine, Brutus dit encore de lui :

il joue et se débauche en folle compagnie.

III, i, 188-189.

ce qui revient à lui attribuer une certaine mollesse, une sensualité qui est, croyons-nous, celle du signe du Cancer.

En relation avec ce signe, signe lunaire, signe d'eau, l'affectivité chez Antoine est aussi très développée; cela se manifestera dans sa fïdélité à la mémoire de (ésar. II représente donc en fait Mercure en Cancer, ce qui, d'après les traités d'astrologie donne une imagination vive, de la bonne volonté, de la fidélité, toutes qualités qui se manifesteront dans son fameux discours. Sa fidélité à la mémoire 
de César, l'habileté qu'il aura de donner connaissance de son testament, seront fatales aux conjurés. Dans son rôle de miroir, il exaltera et magnifiera la gloire posthume du héros bienfaiteur de la Cité atteint de trente-trois blessures $(\mathrm{V}, 1,53$ ), nombre qui s'écrit GL, pour gloria, (ou gloria leonis), chiffre modifié à dessein, puisque Plutarque et Suétone parlaient de vingt-trois blessures.

\section{2-Cassius et Saturne en Capricorne :}

Cassius Longinus Caïus porte un nom maléfique, qui dérive de Cassus (Vide, inutile, à rapprocher de la locution in cassum : en vain $)^{4}$. Le personnage, assez antipathique est chargé, comme Edmond dans Le Roi Lear (I-II, $128 \mathrm{sq})^{5}$, de mettre en question la réalité des influences astrales. Comme on l'a remarqué ce sont les personnages qui contestent l'ordre établi et l'autorité légitime que Shakespeare charge de prononcer de telles paroles. Cassius déclare :

Les hommes sont parfois maitres de leur destin.

La faute est notre fait, non le fait des étoiles

Si nous ne sommes plus que d'infimes esclaves

$$
\text { (underlings) I, ii, 137-139. }
$$

Toutefois l'affirmation est nuancée et rejoint, en l'inversant, l'adage astrologique «les autres inclinent, mais ne déterminent pas». Cassius est probablement le personnage de la pièce le plus nettement typé. Toujours dans la deuxième scène du premier acte, César fait son portrait; s'adressant à Antoine :

Ce Cassius est maigre; il a l'air famélique;

Il pense trop, vois-tu : ces gens sont dangereux $[\cdots]$ je le voudrais plus gras ! Mais je ne le crains pas : $[\cdots]$ homme étique

Il lit beaucoup, observe et son regard vous perce

La conduite des gens : il n'aime les spectacles,

Comme tu fais, Antoine, il n'aime la musique;

Rarement il sourit, ou son sourire a l'air

De se railler lui-même et de se mépriser

D'avoir un moment pu trouver matière à rire

Les hommes tels que lui n'ont point le coeur à l'aise

Tant qu'ils voient s'élever un autre au-dessus 
Et c'est ce qui les rend toujours si dangereux.

d'eux

I, ii, 191-207.

Nous comprenons que c'est le véritable adversaire de César, et il saura gagner son beau-frère (your brother, II, I, 70) Brutus à la cause de la conspiration.

Lorsque Brutus se querelle avec Cassius au quatrième acte, il lui dit :

Par les dieux ! il faut dussiez-vous en crever,

Digérer le venin de votre foie ...[spleen]

IV, iii, 46-47.

Le suicide de Cassius sera typique d'un mélancolique, puisqu'il a mal interprété ce qu'il voyait et a cru à une défaite au moment même d'une victoire. Messala dit alors :

Maudite erreur, enfant de la mélancolie,

$\mathrm{Ah}$ ! pourquoi fais-tu voir à notre esprit sensible

La chose qui n'est point?

V, iii, 67-69.

En outre, le fait que Cassius se suicide le jour même de son anniversaire (This day I breathed first, V, iii, 23), donc alors que le soleil se trouve proche de la position natale, a pour objet de le mettre en relation avec la planète qui gouverne un cycle de 59 ans. La bataille de Philippes ayant eu lieu en 42 avant J.C ceci renvoie aux événements de 100-101 avant J.-C à Rome, marqués par la mort du tribun Saturninus, et la naissance de Jules César.

La parole que prononce Titinus devant son cadavre The sun of Rome is set (V, iii, 63) signifie probabiement qu'il était natif du signe des Gémeaux, qui, traditionnellement, gouverne Rome.

De tout ceci, il résulte que Cassius représente Saturne en domicile dans le signe du Capricorne. A bien des égards, le personnage préfigure Hamlet en qui on a vu, à juste titre, la typification du tempérament mélancolique ou saturnien.

Et son sens de l'amitié, son affection pour Brutus sont à mettre en relation avec un probable Ascendant en Gémeaux

Par ailleurs, il convient de relever la phrase de Brutus. s'adressant à Marc-Antoine :

Mais pour vous

Nos armes n'ont jamais que des pointes de plomb. III, i, 172-173 
La référence au plomp, métal saturnien, désigne en réalité Cassius. Si bien qu'une telle déclaration décrit, en réalité, l'opposition entre Cassius (Saturne en Capricorne) et Antoine (Mercure en Cancer).

\section{3- Casca, sous-Cassius et le signe des poissons-}

Casca, à certains égard, est comme un reflet caricatural de Cassius; il faut probablement entendre, dans son nom, cas et caco, vain et méchant. Brutus dit de lui :

Quel épais compagnon cet homme est devenu !

$$
\text { I, ii, } 292
$$

Mais, comme l'a relevé T.S. Dorsch, il se trouve que "blunt» traduit le nom même de Brutus (hérité de Lucius Junius, Brutus, qui avait feint d'être stupide); ainsi se constitue un subtil jeu d'échos, associant verbalement les conjurés, Brutus, Cassius et Casca.

à Casca :

A la scène iii du même acte (vers 57) Cassius dira votre esprit s'engourdit, Casca (You are dull, Casca)

Il faut connaître le mépris dans lequel Shakespeare semble avoir tenu les natifs du signe des Poissons. Des qualificatifs comme blunt ou dull donnent à penser que Casca se rattache à la lignée détestable qui comprend Clotten (dans Cymbeline) ${ }^{6}$, Cornouailles (dans le Roi Lear) ${ }^{7}$, Caliban (dans la Tempéte) ${ }^{8}$, donc qu'il représente un esprit de lourdeur constamment associé par le dramaturge avec le signe des Poissons.

Le signe des Poissons est en relation avec les pieds de l'homme; or, dans un curieux passage, Casca semble bien s'identifier à son pied :

Tenez, voici ma main :

Conspirez donc pour redresser tous ces abus !

Je veux poser le pied aussi loin que les autres

Et nul plus loin que moi !

(And I will set this foot of mine as far As goes farthest)

I, iii, 118-121.

Rappelons, à ce propos, l'exclamation de Prospero dans la Tempête : 
What I say/my foot my tutor?

1, ii, $471-472$.

Lors de la tentative de révolte de Ferdinand, qu'il a astreint aux tâches manuelles qui reviennent ordinairement à Caliban, représentant dans la pièce du signe des Poissons, et donc des pieds de l'homme.

\section{4- Brutus et Mars en Bélier.}

Cassius dit à son ami Brutus :

On peut, en le forgeant, ployer ton fier métal

(honourable metal)

A ce qu'il répudie.

I, ii, 305-306

Et l'on sait que, dans le fameux discours d'Antoine, l'expression qui reviendra en refrain sera «Mais ce sont des gens honorable». Le métal "honorable» susceptible d'être forgé et aiguisé est la fer; en d'autres termes Brutus représente Mars.

Brutus en proie à l'insomnie monologue :

Depuis que Cassius contre César m'excite (Since Cassius first did whet ${ }^{9}$ me against Caesar) ...

Je n'ai pas fermé l'oeil ...

Entre la prime envie et l'accomplissement

D'un terrible projet, tout l'intérim s'ouvre

Comme la vision d'un hideux cauchemar.

Notre Génie et nos facultés de mortels

Tiennent alors conseil, et cet Etat qu'est l'homme

Comme un petit royaume en de tels moments souffre

En proie à je ne sais quelle insurrection.

$$
\text { II, i, 61-69 }
$$

Ca passage apporte donc la description du microcosme humain en révolution. Le Génie au sens astrologique désigne soit l'étoile, la planète dominante, soit, selon le système de Manilius que Shakespeare connaissait, les aspects bénéfiques du thème associés à la XIe Maison de l'horoscope ${ }^{10}$.

Au moment crucial de la conjuration. Casca tient des propos en apparence futiles :

Là le soleil se lève où je pointe l'épée:

Il gagne en ce moment beaucoup sur le midi

Considérant le jeune essor de la saison. 
Oui, dans deux mois d'ici c'est plus haut vers le nord

Qu'il présente ses feux : l'orient de l'été

Se trouve juste ici, devers le Capitole.

II, i, 106-111.

Ces indications astronomiques ont en réalité pour objet d'indiquer que les Ides de mars sont venues, ce qui sera souligné surabondamment en clair dans les scènes suivantes : C'était la période correspondant à celle du 8 au 15 mars de notre calendrier actuel. Le jour de l'assassinat sera le 15 mars 44 avant notre ère. Il ne fait aucun doute que, dans l'esprit de Shakespeare, le personnage de Brutus s'identifie à Mars en domicile en Bélier et qu'il est en quelque sorte, porteur du fatum, de César (dont il était, peutêtre, le fils).

Au point de vue symbolique, Brutus préfigure Lear qui, comme lui sera associé à Mars en Bélier (Mais Lear revêt en outre, un aspect saturnien $)^{11}$. Brutus se livre à des actions brutales et soudaines d'une manière irréfléchie ou peu réfléchie. La manière dont il s'associe au complot contre César, dont il prend au sérieux le message anonyme «Tu dors, Brutus !», le refus d'un serment liant entre eux les conjurés, la décision d'épargner Marc-Antoine, la permission qu'il donne à celui-ci de prononcer l'oraison funèbre de César, l'ordre d'attaquer donné trop tôt lors d'un engagement décisif, tout souligne la nature monolithique de son caractère. A chaque fois, s'il l'emporte sur Cassius le saturnien, plus lent et plus réfléchi, finalement il se détruit lui-même, et entraîne son complice et ami dans sa chute.

$\mathrm{Au}$ reste, Brutus et Cassius portent l'un et l'autre des noms à consonance défavorable et ils représentent respectivement, Mars et Saturne, planètes généralement considérées comme maléfiques.

Mais il se traduit entre eux une curieuse inversion de fonctions qui peut être mise en relation avec le carré BélierCapricorne (et l'affinité entre deux symboles animaux le Bélier et le Bouc). En effet et spécialement lorsqu'ils entrent en conflit, il apparaít que la valeur que Brutus place en premier est l'honneur tandis que le saturnien Cassius découvre l'importance de l'amitié et de l'affection (love) L'échec même de la conspiration vient de ce que Cassius, par affection pour Brutus, va de concession en concession, 
sur des points essentiels. Le fait d'avoir, contre l'avis de Cassius, laissé vivre Antoine, leur sera fatal.

C'est à propos de la scène de la querelle entre Brutus et Cassius (IV, iii) que S.T. Coleridge déclara :

je ne sais aucun autre endroit de Shakespeare qui suscite à ce point en moi la croyance en la nature surhumaine de son génie que cette scène entre Brutus et Cassius. Selon l'hérésie gnostique il aurait pu être enseigné, avec une absurdité moindre que celle qui se manifeste dans la plupart de leurs dogmes, que l'Etre suprême l'avait chargé de créer les personnages avant même de les mettre en scène (N.9)

Brutus offre des ressemblances avec Macbeth, comme lui marqué par Mars en bélier mais, en outre, victime de l'étoile violente de la Tête de Méduse ${ }^{12}$ : I'un comme l'autre projettent sur le monde la violence et le désordre qui sont dans leur esprit.

L'acte III de la pièce débute par le dialogue entre César et le Devin :

César : Les Ides de Mars sont arrivées

Le Devin : Oui, César, mais elles ne sont point

passées.

et César mourra au vers 77 de cette scène.

En résumé, Brutus conçu comme martien, véritable personnification de la violence, est l'instrument du destin de César, qui s'accomplit aux Ides de Mars.

Lorsque le fantôme de César apparaît à Brutus, il lui dit : "Je suis ton mauvais génie» (IV, iii, 281). Kakodaimon était le nom donné à la VIle Maison de l'horoscope ${ }^{13}$ et César représentant, comme nous allons le voir, le principe solaire, cela veut dire que, dans l'horoscope de Brutus le Soleil est situé en douzième maison (et dans le signe du Lion) ce qui signifie "prison ou exil pour homicide».

Il résulte aussi de la place de la XIle Maison en Lion que l'Ascendant de l'horoscope de Brutus est probablement dans le signe de la Vierge, Edmond dans le Roi Lear sera également associé à la Vierge ${ }^{14}$. Ce signe est souvent considéré par Shakespeare comme maléfique, comme s'il avait une aversion pour l'axe Vierge-Poissons. Toutefois, Miranda, dans la Tempéte représentera l'aspect positif du signe de la Vierge ${ }^{15}$. 


\section{5- Portia et Venus en Sagittaire.}

Portia, femme aimante de Brutus ( $g$ gentle Portia»), (II, , 278 ), en raison même de la complémentarité qu'elle représente par rapport à lui - il ira jusqu'à lui confier le secret de la conjuration - représente le principe vénusien. Mais c'est une Vénus noble et presque guerrière; c'est pourquoi on est conduit à l'associer à un signe de feu, qui ne peut être que celui du Sagittaire, le Bélier étant occupé par Brutus et le Lion, nous le verrons bientôt, revenant à César. Vénus dans ce signe peut conduire à certaines aberrations et en effet Portia se biesse elle-même à la cuisse (région du corps qui correspond précisément au Sagittaire), pour prouver son courage physique (II, i, 300-301). Elle se suicidera en avalant $d u$ feu (IV, iii, 156), comme le rapporte en effet Plutarque : elle placera dans sa bouche des charbons ardents.

Il faut relever que Shakespeare met volontiers les personnages sacrifiés en relation avec le Sagittaire : Othello est marqué par Antarès, étoile de la région du Sagittaire ${ }^{16}$; Duncan dans Macbeth ${ }^{17}$ et le Fou dans Le Roi Lear ${ }^{18}$ sont également des représentants de ce signe. Il y a là un mécanisme de répétition assez curieux qui n'est peut-être pas entièrement volontaire et qui peut être mis en relation avec la place du Soleil dans l'horoscope du poète, en Taureau ${ }^{19}$ : car si l'on compte à partir du Taureau, le Sagittaire est le huitième signe, correspondant analogiquement à la VIlle Maison de l'horoscope ou Maison de la Mort.

Les signes de feu

Quoi qu'il en soit, la triplicité de feu revêt dans Jules César une particulière importance : le trigone BélierSagittaire décrit la relation conjugale entre Brutus et Portia et le trigone Bélier-Lion correspond à la probable filiation César-Brutus. D'après Suétone le mot de César mourant prononcé en grec fut : "Kai $\sigma u ́, z \varepsilon k$ kov.». Traditionnellement les trois signes royaux sont les signes de feu même si Shakespeare, parce qu'il était natif du Taureau avait pris l'habitude de considérer ce signe comme le signe royal (ce qui fut vrai, par exemple à l'époque mycénienne dans 
l'Egée).

Brutus, s'efforçant de justifier l'assassinat de César emploiera une image révélatrice :

Notre pitié pour le malheur public de Rome,

Comme un feu chasse un feu - chassant notre

Pour César, frappe encor par pitié.

pitiẻ

III, i, 169-170-172.

En somme, le feu du Bélier (identifié à la pitié pour le malheur public éprouvée par Brutus et ses associés ) a mis à mort le feu solaire du Lion.

Le corps de César est brûlé par une populace déchainée (III, ii, 258); celui de Brutus sera incinéré par ses ennemis $(\mathrm{V}, \mathrm{v}, 55)$.

\section{6- Jules Cesar ou le soleil en lion.}

Le nom de César d'après les étymologistes dérive de circonstances exceptionnelles de sa naissance : Pline et Nonius Marcellus l'expliquent a caeso matris utero; Festus dit caesares dicti quia caesa matre nascuntur ${ }^{20}$.

Parmi les prodiges annonciateurs de la mort de César que rapporte Casca à la scène iii du premier acte il faut relever :

j'ai près du Capitole un lion rencontré,

Qui, le regard luisant, farouchement passa

Sans plus m'inquiéter.

1. iii, 20-22.

Lorsqu'on rapporte à César les événements prodigieux de la nuit, il déclare :

\section{Peut-on rien éviter}

De ce qu'a décrété la puissance des dieux ?

$$
\text { II, ii, 26-27. }
$$

Et dans la suite d'un discours où éclate une fatuité destinée, pour une part à rendre acceptable au spectateur la scène de l'assassinat, il affirme :

[...] Le danger sait fort bien

Que César est encor plus dangeureux que lui :

Nous sommes deux lions mis bas le même jour;

Et moi je suis l'ainé, je suis le plus terrible.

III, ii, 44-47.

En cet endroit (ésar, né le 12 juillét 100 s'identifie donc 
expressément au soleil en domicile dans le signe zodiacal du Lion. Au reste, dès le premier acte, Cassius avait, à deux reprises, comparé César à un lion :

Maintenant, je pourrais te signaler un homme

Semblable de tout point à cette horrible nuit :

Il lance des éclairs, il tonne, ouvre les tombes,

Rugit tel le lion qu'on garde au Capitole.

Et, dans la même scène, il disait de César :

I, iii, 72-75.

S'il est lion, c'est que nous sommes faons craintif's

I, iii, 106.

et il poursuivait en évoquant Rome transformée en brasier pour illuminer César.

On s'étonne parfois que la tragédie soit intitulée Jules César, alors que le véritable héros en serait Brutus. Mais le sujet de la pièce est, pour une part importante, la survie et la vengeance posthume de César, dont le fantôme apparaît à plusieurs reprises à ses assassins.

Il n'est pas hors de propos de rappeler ici les paroles que le poète Ovide prête à la déesse Vesta, dans les Fastes (III, 697-710) :

je m'apprêtais à passer sous silence les glaives qui tuèrent le prince, lorsque Vesta, de son chaste foyer s'exprima ainsi «N'hésite pas à les rappeler; c'était mon prétre, c'est moi que ces mains sacrilèges frappèrent avec le fer. Je l'enlevais et ne laissais dernière lui que son apparence; ce qui tomba par l'épée était l'ombre de César.

Transporté au ciel il vit le sanctuaire de Jupiter et il possède dans le grand Forum un temple qui lui est consacré. Mais tous les impies audacieux qui, défiant la volonté des dieux, profanèrent la tête du Pontife, sont couchés dans la mort, la mort qu'ils méritaient. En portent témoignage Philippes et ceux dont les os blanchissent le sol. Tel fut l'oeuvre de César (Auguste), son devoir; venger son père par de justes armes.

Nous croyons que, dans l'esprit de Shakespeare, il ne faisait aucun doute que les événements de Macédoine faisaient partie intégrante de l'histoire de Jules César, que celle-ci, sur le plan humain, ne prenait fin qu'à la bataille de 
Philippes. La place centrale que César ne cesse d'occuper dans la pièce se trouve soulignée par le fait, que juste avant d'être assassiné, pour expliquer à Cassius qu'il ne peut gracier Publius Cimber, il se compare lui-même à l'étoile polaire :

But I am constant as the the northern star III, i, 60. Et le milieu même de la tragédie, si on compte les lignes dans l'édition Arden, correspond au retournement de situation qui résulte du fait que Brutus autorise MarcAntoine à prendre la parole et Cassius dit alors : avec une juste prémonition de la suite des événements :

I know not what may fall : I like it not.

III, i, 243.

Je ne sais ce qui peut advenir, cela ne me plait pas Il est d'ailleurs remarquable que, pour que le meurtre de César devienne acceptable aux yeux de Brutus, il a eu besoin de l'envisager comme un sacrifice sanglant. Et il déplore d'être obligé de verser le sang pour tuer l'esprit (II, i, 168-170). Une idée fausse analogue s'emparera de l'imagination d'Othello, au moment où il décidera de tuer Desdémone (Mais il s'agira alors d'un sacrifice non-sanglant, prenant la forme de l'étouffement). Toute une symbolique du sang. mis en relation avec le feu, la lumière, l'or solaire, et, qui, pour une part, vient directement de Plutarque, se trouve associée au personnage de Jules César et doit croyons-nous, étre rapprochée du fait que le personnage représente le Soleil, en domicile dans le signe du Lion. Plusieurs critiques ont vu l'importance de ce groupe d'images, sans pour autant en discerner les implications astrologiques. C'est le cas en particulier de G. WilsonKnight, dans les deux essais sur Jules César qu'on trouve dans son livre The Imperial Theme. Ce critique a vu aussi que, dans la pièce le coeur (autre symbole du Lion), est constamment mis en relation avec l'or : c'est ainsi que Brutus parle de "monnayer son coeur" et de "changer goutte à goutte, son sang en drachmes" (IV, iii, 72-73).

D'autre part, de nombreuses références aux yeux, à la vision, renvoient égaiement à la symbolique de la lumière, cela de manière d'autant plus certaine que, dans les Hiéroglvphes d'Horapollo. Dieu est écrit par l'hiéroglyphe du Soleil, Râ. 
Nous avons rappelé que la mise à mort du héros solaire était annoncée par des prodiges : signes dans le ciel, comète, animaux sauvages se promenant en liberté dans la Ville, morts qui ressuscitent. Tout cela était dans Plutarque. Il sera question des 33 blessures de César $(\mathrm{V}, \mathrm{i}, 53)$ ce nombre, avons-nous dit, écrit L.G. pour Leo Gloriosus mais c'est aussi le triple de 11 , nombre solaire.

Le sang de César répandu constitue l'image centrate de la pièce, comme le sang de Duncan et la Tête de Méduse dans Macbeth.

Les mots de la famille de sang, très nombreux, répandent sur toute la pièce une lueur écarlate. Le relevé des passages importants à cet égard avait été fait par $G$. Wilson Knight (op. cit. p. 45 sq., "The torch of life»).

M. Spevack dans The Harvard Concordance to Shakespeare relève dans Jules César 24 occurrences de blood (contre 23 dans Macbeth) et 10 de bloody (14 dans Macbeth).

Le sang apparaît déjà dans le rêve prophétique de Calpurnia :

En rêve, cette nuit, elle a vu ma statue :

Du sang pur en coulait comme d'une fontaine

Par centaines de jets : et des Romains en nombre,

Le sourire à la lèvre, $y$ venaient se baigner [...]"

II, ii, 76-79.

Décius donne de ce réve une interprétation favorable selon laquelle il voudrait dire :

... qu'en César la grande Rome puise

Un sang rénovateur.

II, ii, 87-88.

Comme l'a relevé Léo Kirschbaum ${ }^{21}$, l'horrible rituel des conjurés trempant leurs mains dans le sang de César, déjà décrit dans le rêve de Calpurnia, n'était pas dans le récit de Plutarque; c'est, semble-t-il, une invention de Shakespeare :

Alors baissons

Nos fronts, baignons nos bras dans le sang de

César

Jusqu'au coude ...

III, i, 105-107.

Puis vient cette étonnante évocation scénique :

Que de fois on verra saigner sur les théâtres 
Jules César gisant aux pieds du grand Pompée...

III, i, 114-115.

Dans sa douleur, Antoine s'adresse au cadavre de César, et dans la suite de la même scène, en quelques vers on trouve : "Quel sang reste à verser (152)" Le plus noble sang de tout cet univers" (156) et aussi "whilst your purpled hands do reek and smoke» (158).

Dans la réponse de Brutus, on relève "we must appear bloody and cruel» (165); «bleeding business» (168).

Rendant hommage à la mémoire de César, Antoine toujours dans la suite de la même scène dit :

Si j'avais autant d'yeux que ton corps a de plaies,

Versant autant de pleurs qu'elles versent de sang 200-201

Une telle image établit de lien entre le registre de la lumière et celui du sang.

Et lorsqu'il restera seul en présence du cadavre, Antoine dira encore :

O pardonne-le moi saignant amas d'argile (254) [...]

Malheur au bras qui put verser un sang si riche ! En cette heure, je le prédis sur ces blessures, Bouches sans voix, ouvrant leurs lèvres de rubis...

257-260.

Le sang de César est presque divin, il avait une valeur infinie. Et c'est pourquoi, pour expier leur crime, les conspirateurs devront tous périr. A la scène suivante, Marc Antoine, dans son célèbre discours devant le corps de César, fera encore quatre références à son sang et à ses blessures (III, ii, $137-138 / 134-135 ; 182 / 180 ; 229 / 227)^{22}$.

Dans toute la pièce, le sang est concu comme le support de l'esprit et de l'honneur (II, i, 136); il est positivement apparenté à la lumière. En d'autres termes, l'oeuvre de Shakespeare véhicule en ce cas un enseignement très proche de celui des Védas.

Toute cette symbolique culminera dans un passage sur lequel nous reviendrons; dans lequel Marc Antoine fait de César le cœur même de l'univers :

O world, thou wast the forest to this hart And this, indeed, $\mathrm{O}$ world the heart of thee III, i, 207-208. Un autre passage de l'un des discours d'Antoine mérite de 
retenir l'attention, c'est celui où il déclare :

For Brutus, as you know, was Ceasar's angel

III, ii, 185.

Le mot angel ne doit pas en cet endroit être pris dans le sens du grec daimon : il a probablement pour objet de souligner l'importance de la planète Mars dans l'horoscope de César, puisque, ainsi que nous l'avons vu, Brutus, dans cette tragédie est le représentant de cette planète.

On peut même se demander si cela ne signifie pas que Shakespeare considérait que, dans le thème de la nativité de César, Mars se trouvait en Bélier, ce qui signifierait dans son cas une brillante carrière militaire. Allant plus loin encore dans le sens de cette hypothèse ne faut-il pas imaginer que Mars en Bélier marquerait l'hérédité de Brutus, fils de César, et que la structure même de la pièce, et la conception des personnages reposerait sur un horoscope supposé de César ? Sans entreprendre de résumer ce qui a été indiqué par Stefan Weinstock dans son Divus Julius (Oxford 1971) il faut souligner que le processus de déification de Jules César conduisait, inévitablement, à l'identifier au Soleil. Dès lors, la seule position acceptable du Soleil dans l'horoscope était bien en domicile dans le signe du Lion.

$\mathrm{La}$ nature théoriquement «léonine» de Jules César se trouve, en quelque sorte, confirmée par le fait qu'à l'instigation de l'astronome Sosigène, il imposa le calendrier solaire, dit calendrier Julien.

\section{7- Calpurnia : La Lune en Verseau}

Calpurnia (Bonne et Pure ?), miroir parfait, en raison même du principe de complémentarité des opposés représente la Lune dans le signe du Verseau, ce qui prédispose au mysticisme et aux visions. Et, en effet, son principal rôle dans la pièce est (II, ii) d'essayer de dissuader César de se rendre au Sénat en ce 15 mars, en raison du terrible rêve prémonitoire qu'elle a eu dans la nuit. Mais nous l'avons rappelé, Décius, survenant, donne du rêve en question une interprétation favorable qui renverse la décision de César et causera sa perte.

8- Octave : Jupiter en Balance.

On n’a jamais interprété correctement, semble-t-il, 
un passage, déjà brièvement mentionné, essentiel pour notre propos et où les commentateurs ne voient qu'un jeu de mot un peu précieux et assez banal sur hart et heart. Certains, comme S.T. Coleridge sont allés jusqu'à contester l'attribution des vers en question à Shakespeare ${ }^{23}$. S'adressant au cadavre de César, Antoine dit :

Pardonne, Julius ! C'est ici, brave cerf, [hart]

Que, forcé, tu tombas, et voici tes chasseurs !

Tout souillés de curée et rougis de carnage

Tu étais univers la forêt de ce cerf, (Et César, certes ô monde était ton coeur même $)^{24}$

Maintenant, comme un daim, dagué de plusieurs (heart)

Tu gis là, sous nos yeux. princes

III, i, 204-210. Nous avons déjà relevé dans ces vers l'image, essentielle, du coeur (que la traduction doit évidemment conserver). Il reste à expliquer l'alternance heart/hart.

- Heart : désigne le signe du Lion, domicile du Soleil en relation symbolique avec le coeur de l'homme.

- Hart : le cerf est un très ancien symbole de l'automne, (après avoir sans doute représenté le soleil levant), il renvoie au signe de la Balance ${ }^{25}$.

Il apparait d'abord que la Balance était le signe de la conception de César, et on sait que les Anciens accordaient autant d'importance au signe de la conception qu'à celui de la naissance.

Mais, en réalité, c'est Octave, qui succèdera à César et deviendra Auguste, qui est représenté par le Cerf. Plus précisément, Octave représente Jupiter en Balance (ce qui signifie "amitié entre gens de haute situation", description de son alliance avec Antoine).

Ainsi l'alternance heart-/hart qui traduit l'altemance latine Cor-cordis/Cornu-cornus) décrit aussi le fait que le coeur-cerf mis à mort revivra dans son fils adoptif Octave. Remarquons d'ailleurs que l'Arcane VIII du Tarot. $L a$ Justice, qui renvoie au nom d'Octave est précisément en relation avec le signe de la Balance, (puisque la halance est l'attribut de Thémis).

Nos déductions sont confirmées par deux passages du 
livre IV des Astronomica de Manilius, dans le premier on lit :

Quand les pinces de l'automne commencent à se lever, il est béni celui qui est né sous l'équilibre de la Balance. Juge il établira une Balance qui pèsera la vie et la mort; il imposera le poids de son autorité et fera des lois. Les villes et les royaumes trembleront devant lui et seront gouvernés par un seul commandement, tandis qu'après son séjour sur terre l'administration du ciel lui incombera.

(IV, 547-552)

Les commentateurs indiquent en cet endroit qu'il s'agit bien d'Auguste, né le 22 septembre 63 avant J.C. ${ }^{26}$

Un second passage du même livre des Astronomica dit d'ailleurs :

L'Italie appartient à la Balance, son véritable signe; c'est sous lui que furent fondées Rome et sa souveraineté sur le monde, Rome qui contrôle le devenir des choses, élevant ou abaissant les nations placées sur les plateaux de la Balance.

(IV, 773-775)

A propos de l'horoscope d'Auguste, il convient aussi de citer une page célèbre de Suétone.

Durant sa retraite à Appollonie, Auguste était monté, en compagnie d'Agrippa, à l'observatoire de l'astrologue Théogène, Agrippa le consulta le premier, mais, comme Théogène lui faisait des prédictions magnifiques, presque impossibles à croire, Auguste se refusait obstinément à lui fournir des indications sur sa propre naissance, de peur d'être humilié par des révélations moins brillantes. Lorsque, après maintes prières, il y eut consenti, en hésitant, Théogène s'élança de son siège et se prosterna devant lui. Plus tard, Auguste eut une si grande confiance dans ses destinées qu'il fit publier son horoscope et frapper des pièces d'argent portant le signe du Capricorne, sous lequel il naquit .

(Suétone : Dirus Augustus, \& XCIV, Vies des Douze Césars, t. I, trad. Henri Ailloud, P. Belles Lettres, 1931). 
Les célèbres monnaies à l'effigie du Capricorne représentaient le signe de la conception d'Auguste.

En marge de la tragédie de Shakespeare, on peut rappeler un autre passage de Suétone :

A Philippes, un Thessalien lui prédit la victoire, de la part du divin César, dont le fantôme s'était présenté à lui dans un chemin écarté .

(Ibid, $\S \mathrm{XCVI}$ )

\section{Les quatre temperaments.}

Si on envisage le système constitué par Shakespeare à travers les personnages de la tragédie, on constaste qu'il y associe et oppose les représentants des deux maléfiques, Saturne et Mars ce sont Cassius et Brutus, chefs des meurtriers de César; tandis que les deux vengeurs Antoine et Octave sont associés respectivement à Mercure et à Jupiter. Il apparaít d'ailleurs que le poète n'a pas connu en détail le thème de nativité d'Octave Auguste car, dans ce cas, il aurait probablement interverti l'attribution des planètes bénéfiques (Jupiter et Mercure). Il s'est semble-t-il, contenté de l'indication selon laquelle le futur empereur était natif du signe de la Balance.

Le système épouse donc les axes des équinoxes et des solstices et s'établit ainsi :

\section{CASSIUS \\ (Saturne en Capricorne) \\ Mélancolique}

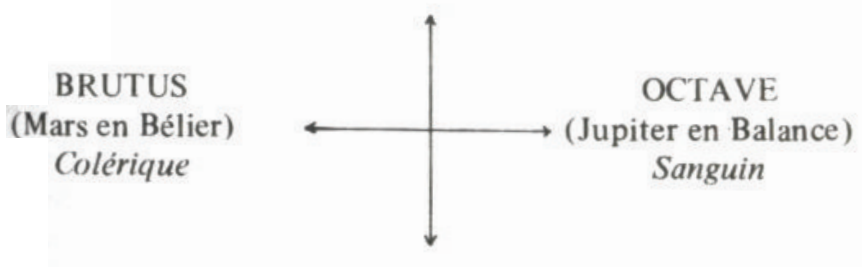

ANTOINE

(Mercure en Cancer)

Flegmatique 
Comme nous l'avons précédémment établi, un tel quatuor se retrouve dans Le Roi Lear : il n'est pas sans intérêt d'indiquer ici les correspondances qu'on peut établir :

\begin{tabular}{|c|c|c|c|c|}
\hline \multirow{2}{*}{$\begin{array}{l}\text { Signe du } \\
\text { Zodiaque }\end{array}$} & \multirow[t]{2}{*}{ Elément } & \multirow[t]{2}{*}{ Tempérament } & \multicolumn{2}{|c|}{ Personnages } \\
\hline & & & de Jules César & du Roi Lear \\
\hline Bélier & Feu & Colérique & Brutus & Lear \\
\hline Cancer & Eau & Flegmatique & Antoine & Regane \\
\hline Balance & Air & Sanguin & Octave & Albany \\
\hline Capricorne & Terre & Mélancolique & Cassius & Cloucester \\
\hline
\end{tabular}

Comme nous avions essayé de l'établir de façon détaillée, dans Le roi Lear, les éléments jouent tous un rôle. Dans Jules César domine la triplicité du feu, et toutes les images qui s'y rapportent. D'autre part, la symbolique des métaux, en relation avec les planètes contribue à donner à la pièce sa tonalité particulière.

10- La série planétaire.

Si nous examinons comment se constitue dans la pièce la série planétaire nous trouvons, en suivant l'ordre des jours de la semaine :

\begin{tabular}{|l|c|r|}
\hline CESAR & Le soleil en domicile en Lion & L'or \\
\hline CALPURNIA & La Lune en Verseau & L'argent \\
\hline BRUTUS & Mars en domicile en Bélier & le fer \\
\hline ANTOINE & Mercure en Cancer & Le mercure \\
\hline OCTAVE & Jupiter en Balance & L'étain \\
\hline PORTIA & Vénus en Sagittaire & Le cuivre \\
\hline CASSIUS & Saturne en domicile en Capricorne & Le plomb \\
\hline
\end{tabular}


Les sept métaux ne sont pas effectivement nommés dans la pièce, mais on y relève des références plus ou moins explicites à quatre d'entre eux l'or, le fer, le mercure et le plomb.

Trois personnages sont décrits par des planètes en domicile, ce sont César, Brutus et Cassius. C'est une manière de souligner qu'ils sont les plus importants. En ce qui concerne Antoine, Mercure n'est pas en domicile : le personnage posait en quelque sorte le problème de la "quadrature du septénaire», puisqu'il s'agissait, en même temps de faire apparaître les quatre tempéraments.

Dans la majorité des cas, le caractère même des personnages renvoie à un signe zodiacal. Nous avons en outre relevé, dans trois cas, d'intéressantes confirmations en relation avec la symbolique zodiacale des parties du corps humain : César représente le coeur du monde (Lion); Portia se blesse volontairement à la cuisse (Sagittaire); Casca s'identifie à son pied (Poissons).

En comparant par exemple Jules César au Roi Lear ou à La Tempête, on voit que, dans l'apparente rigidité du cadre astrologique, il suffit de faire varier l'âge, le sexe des personnages, la répartition des planètes dans les signes, les conditions sociales, pour obtenir une variété qui est le reflet même de celle de la vie. En même temps, la connaissance du substrat astrologique de la pièce donne toute leur saveur et leur pleine portée aux nombreuses images et métaphores, qui constituent en réalité un système rigoureux, car loin d'être, comme on l'a parfois prétendu, arbitraires ou incohérentes, elles ont valeur d'intersignes et sont étroitement liées, tant à la conception des personnages qu'au déroulement de l'action.

A notre avis la présence d'une symbolique astrologique aussi élaborée dans Jules César suffirait à prouver que l'auteur de cette tragédie est bien aussi celui qui a écrit Macbeth, Othello, Le Roi Lear et La Tempête.

Le point de départ de la pièce comme souvent dans le théâtre de Shakespeare, a pu être une méditation sur les noms de César, Brutus et Cassius, conçus à partir de la lecture de Plutarque comme des représentants du Soleil, de Mars et de Saturne. 


\section{NOTES}

1. Julius Caesar. The Arden Shakespeare, Londres, Methuen, 1955 (1977), introd. de T.S. Dorsch, p. LXVII.

2. Il ne faut pas perdre de vue que Plutarque était prêtre de Delphes et qu'il a pu inclure à dessein dans ses récits d'événements historiques quantité de détails symboliques que Shakespeare n'a fait que reprendre, si bien qu'on se trouve en présence d'une triple symbo. lisation : à une réalité historique elle-même pleine de signes se superposent les apports de Plutarque, puis l'interprétation de Shakespeare Voir par exemple le passage des 23 blessures de César selon Plutarque aux 33 blessures dans la tragédie.

3. Nous citons le texte en français d'après la traduction de Charles Marie Garnier; coll. Shakespeare, Les Belles Lettres, 1929 (1967); chaque fois que cela est utile, nous citons quelques passages de l'original anglais.

4. Voir A. Ernout et A. Meillet : Dictionnaire étymologique de la langue latine, à l'article «cassus».

5. Voir : J. Dauphiné et J. Richer : Les Structures symbo. liques du «Roi Lear» de Shakespeare, P.. Belles Lettres, 1979, §21 (Titre abrégé ci-après en Struct. symb.R.L.).

6. Voir l'article de James Dauphiné : Le Secret de Cymbeline, Etudes anglaises, 1979.

7. Struct, Symb, R,L., §26.

8. Voir de J. Richer «La conception des personnages dans «L a Tempête de Shakespeare et la structure de la pièce». Annales de la Faculté des Lettres de Nice, no 34, 1978 (Hommage à Emile Gasquet).pp. 77-86.

9. To whet signifiant «aiguiser», on retrouve ici une allusion au fer, métal «noble».

10. A. Bouché-Leclercq : L'astrologie grecque, p. 1899; (réimp. Bruxelles, 1963 ), p. 280 et Manilius : Astronamíca, 2, 888 sq. $\mathrm{L}$ a onziè $\mathrm{me} \mathrm{m}$ aison est no $\mathrm{mm}$ ée Agathos daimon ou Bonus genius.

11. Struct, symb, R.L. $\oint 12,13$ et 14.

12. Cf. J. Richer: Prestiges et damnation dans le thédre de Shakespeare, $1,1,2,3$

13. A. Bouché Leclercq : op. cit, loc cit. et Manilius : Astronomica, 2, 865 sq. La douzième maison est appelée $K a k o s$ daimon ou Malus genius.
14. Struct symb.R.L. $\S$ is.
15. Article cité ci-dessus n.8. 
16. J. Richer : Prestiges et damnation ..., $11,15$.

17. Ibid., $\oint 6$.

18. Struct. symb.R.L. $\oint 18$

19. Ibid.. $\oint 20$ et $\oint 33$.

20. A. Ernout et A. Meillet: Dictionnaire étymologique de la langue latine, article «caedo». Selon Pline $(7,47)$ et Nonius Marcellus (Ed. W. M. Lindsay 566, 26), Caesar est expliqué par «a caeso matris utero» ou «de l'utérus coupé de sa mèren et l'on trouve dans Festus $(50,7)$ "caesares dicti quia caesa matri nascuntur»: «ils sont dits César parce qu'ils naissent d'une mère coupée».

21. Léo Kirschbaum : «Shakespeare's stage blood», P, M.L. A. 1949: reproduit par Peter Ure dans Julius Ceasar. «Casebook series». $1969,(1975)$.

22. Les vers ne portant pas les mémes numéros dans les éditions utilisées nous donnons d'abord le numéro de l'édition des Belles Lettres, suivi de celui de l'édition Arden.

23. En effet, faute d'avoir saisi le sens profond de l'alternance heart/hart, S.T. Colerigdge prétendait qu'il s'agissait d'une inter. polation non-shakespearienne. Et, à l'appui de cette thèse, il affirmait qu'on ne trouvait rien de semblable en d'autres endroits de son oeuvre. Or, nous avons, en particulier, montré que dans Le Roi Lear l'opposition de la native du Lion bienveillante (Cordélie) au vil natif des Poissons (Cornouailles) se traduisait par l'opposition de cor à corn. (Corn pouvant aussi se référer à Edmond, natif de la Vierge). Dautre part, dans la même pièce, le remplacement de Lear par Cornouailles correspondant à la substitution de corn à crown. Enfin la complémentarité Cordélie-Kent se marque par le fait que Douvres, qui joue un rôle si important dans la pièce est située précisément dans le Kent. (Sur tout cela voir Struct symb. R.L. spécialement $\oint 16,26$ et 31 ).

24. "And this indeed, $O$ world, the heart of thee», nous traduisons.

25. Voir notre Géographie sacrée du monde grec, 1974. p. 154 et p. 196 et notre article sur «L'ancien calendrier chypriote et la géographie sacrée de I'lle de Chypre», L'Astrologue, $N=45.46$, ler sem. 1979,pp.71-83.

26. Manilius: Astronomica, éd. G.P. Goold, The Loeb classical Library, 1977 , n.p.266.

27. Struct, symh, R.I. $\oint 32$ et tableau p. 113. 